\title{
Increasing the treatment motivation of patients with somatic symptom disorder: applying the URICA-S scale
}

\author{
Johannes Mander ${ }^{1,2,4^{*}} \mathbb{D}$, Georg Schaller ${ }^{1}$, Hinrich Bents ${ }^{1}$, Ulrike Dinger ${ }^{3}$, Stephan Zipfel ${ }^{2}$ and Florian Junne ${ }^{2}$
}

\begin{abstract}
Background: Therapeutic intervention programs for somatic symptom disorder (SSD) show only small-to-moderate effect sizes. These effects are partly explained by the motivational problems of SSD patients. Hence, fostering treatment motivation could increase treatment success. One central aspect in SSD patients might be damage to motivation because of symptomatic relapses. Consequently, the aim of the present study was to investigate associations between motivational relapse struggle and therapeutic outcome in SSD patients.

Methods: We assessed 84 inpatients diagnosed with SSD in the early, middle and late stages of their inpatient treatment. The maintenance subscale of the University of Rhode Island Change Assessment-Short (URICA-S) was applied as a measure to assess motivational relapse struggle. Additionally, patients completed measures of treatment outcome that focus on clinical symptoms, stress levels and interpersonal functioning.

Results: The results from multiple regression analyses indicate that higher URICA-S maintenance scores assessed in early stages of inpatient treatment were related to more negative treatment outcomes in SSD patients.

Conclusions: SSD patients with ambivalent treatment motivation may fail in their struggle against relapse over the course of therapy. The URICA-S maintenance score assessed at therapy admission facilitated early identification of SSD patients who are at greater risk of relapse. Future studies should incorporate randomized controlled trials to investigate whether this subgroup could benefit from motivational interventions that address relapse.
\end{abstract}

\section{Background}

Somatic symptom disorder (SSD) is characterized by physical symptoms that cannot be fully explained medically. The prevalence rates of SSD are high, ranging from 5 to $7 \%$ of the population [1]. Longitudinal studies have demonstrated significant chronicity, with up to $90 \%$ of cases lasting longer than 5 years [2,3]. SSD symptoms are often associated with severe impairments in the general functioning of everyday life, substantial psychosocial disabilities and diminished quality of life [3, 4]. These patients show disproportionately elevated rates of medical care utilization and account for at least $25 \%$ of all doctor visits in primary care $[5,6]$, leading to high health care

\footnotetext{
* Correspondence: johannes.mander@zpp.uni-hd.de

${ }^{1}$ Center for Psychological Psychotherapy, University of Heidelberg, Heidelberg, Germany

2Department of Psychosomatic Medicine and Psychotherapy, Medical

University Hospital of Tübingen, Tübingen, Germany

Full list of author information is available at the end of the article
}

costs [7]. Consequently, psychotherapeutic treatments are crucial [8]. However, recent meta-analyses have demonstrated that therapeutic interventions for SSD patients yield only small-to-moderate effect sizes [7, 8]. Therefore, a better understanding of individual patient needs is important, as this could help to develop better treatments [9]. One core struggle among SSD patients is their highly ambivalent motivation towards participation in psychological treatments due to their usually somatically focused health beliefs [7]. More specifically, patients often search for the correct medical treatment for their problem [5], which can negatively impact treatment motivation for psychological interventions [10]. A better understanding of the motivation to change is thus critical to improving psychotherapeutic treatments for SSD [8].

The transtheoretical model (TTM; $[11,12])$ offers a comprehensive theoretical framework for treatment motivation and has been investigated empirically in several studies [13]. The TTM is based on an integrative analysis 
of theories of behavior change from the most influential therapy schools [11] as well as on empirical studies on motivational change cycles of self-helpers with different health behaviors (e.g., smoking cessation) [14]. The model has been further improved by analyzing treatment motivation in psychotherapy patients [13], which suggests that therapeutic interventions should be adapted to match the motivational stage of the individual patient [11]. Based on the aforementioned empirical findings, the TTM distinguishes five motivational stages of change: In the precontemplation stage, patients have no desire for therapeutic change. In the contemplation stage, ambivalence about therapeutic change is central. In the preparation stage, there is commitment to therapeutic change. In the action stage, the focus is on actively working on therapeutic change, and in the maintenance stage, patients focus on relapse prevention.

It is of relevance to note that maintenance does not imply that patients have already passed through psychotherapy. Instead, it implies they have relapse struggles when trying to solve their problems. This applies to both the time in psychotherapy and the time before entering psychotherapy $[11,13]$. According to the TTM, patients move through the stages of change in a spiral pattern. More specifically, this means that relapses are considered to be integral parts in the change cycle [13]. Recycling to an earlier stage of change provides patients with the opportunity to use different, more effective change strategies [11]. Further, the stages of change are operationalized using a dimensional approach [11]. This dimensional approach conceptualizes the stages of change as a multidimensional construct in which each patient has specific values in all the stages of change at the same measuring time [15]. The most widely applied dimensional measure for assessing the stages of change is the University of Rhode Island Change Assessment (URICA, $[16,17])$. The URICA consists of 32 items that include eight items for each of the four subscales, precontemplation, contemplation, action and maintenance. A meta-analysis identified a medium effect size of $\mathrm{d}=.46$ to describe the predictive effects of the stages of change, as measured by the URICA, on therapeutic outcome [13]. The URICA-S, a 16 -item short version with excellent psychometric properties, has also been validated recently $[18,19]$. It should be noted that the URICA-S maintenance scale includes several items that characterize struggles with relapse as being a consequence of ambivalent treatment motivation $[18,20]$.

Given the lack of empirical evidence on the motivational change stages in SSD using the URICA-S, research findings from anorexia nervosa (AN) patient groups may provide ideas about how to investigate the stages of change in SSD. In AN patients, for instance, the URICA-S maintenance scores at the beginning of treatment were helpful in identifying patients who experienced less symptom change across the course of inpatient therapy [21]. Consequently, the authors concluded that patients with higher scores on the maintenance domain of the URICA-S could be a subgroup in need of additional treatment elements to combat ambivalence and prevent relapse. In fact, initial empirical evidence suggests that this AN subgroup benefits from additional treatment components, such as emotion regulation and skills training related to drawbacks, additional psychoeducation, as well as aftercare programs following inpatient therapy [22].

These findings from the AN patient groups could potentially apply to SSD patients as well because relapse struggle is a typical phenomenon in those patients too $[5,10]$. More specifically, many SSD patients have a long history of medical treatment before entering psychotherapy $[2,3]$. These treatments sometimes result in shortterm success, followed by a relapse of the problems [10]. These mostly unsuccessful attempts to address their problems before entering psychotherapy can be described in the spiral pattern of the TTM: some SSD patients try to resolve their problem (e.g., with medical treatments), have some short-term success, but then fail to maintain success and consequently relapse $[10,18]$. This can also often be related to feelings of frustration and, subsequently, to decreased motivation in treatment [9]. This subgroup of SSD patients might need additional treatment components, such as interventions pertaining to emotion regulation strategies and skills training related to drawbacks, all of which could help these individuals cope with their relapse struggle. Therefore, it would be helpful to develop a stages of change measure that could facilitate the early identification of SSD patients who are at risk of suffering from motivational and relapse difficulties. For instance, although the clinical relevance of the Pain Stages of Change Questionnaire (PSOCQ; [23, 24]), a disorder-specific stages of change measure for SSD patients, has been established [10,25], this measure has no subscale that directly assesses relapse struggle (detailed discussion in: [18]). The PSOCQ maintenance score reflects the ability to maintain progress $[10,22]$. Hence, the URICA-S maintenance scale could help identify subgroups of SSD patients who need special therapeutic attention.

As mentioned above, similar to the initial empirical evidence obtained in a sample of AN patients [22], SSD patients with high URICA-S maintenance scores might need specific treatment elements to address motivational relapse struggle. As such, the URICA-S maintenance score could be applied as one component of a relapse identification tool for SSD patients. More specifically, it could be applied to the identification of subgroups of SSD patients who could specifically benefit from motivational interventions that address relapse. However, we must first obtain empirical evidence that the URICA-S maintenance scale can indeed identify SSD patients who 
are at risk of relapse. In this context, future studies should examine the extent to which therapeutic treatment can be improved using the URICA-S in SSD patients. The purpose of the current study, therefore, was to address this research gap. Based on the results of Mander et al. [21] in AN patients, we hypothesized that higher maintenance scores in the early, middle and late stages of therapy would be associated with more negative treatment outcomes in SSD inpatients. We specifically focused on three different relevant outcomes for SSD patients: general clinical symptoms, general stress level and interpersonal functioning.

\section{Method}

\section{Treatment and study design}

All patients completed an inpatient psychotherapy program at the Department of Psychosomatic Medicine and Psychotherapy of Tuebingen University, Germany. The treatment consisted of individual therapy, group therapy, art therapy and music therapy twice per week. Psychotherapy incorporated a cognitive-behavioral approach with supplementary interpersonal psychotherapeutic elements. Eighteen psychotherapists (15 female) with at least 1 year of experience conducted therapy. Individual therapy was conducted two times per week. Inpatient treatment ranged from a minimum of 14 days to a maximum of 64 days, with a mean of 38.1 (SD 14.2) days.

Patients were assessed at baseline $\left(t_{1}\right)$, after the 8th session $\left(t_{2}\right)$ and after the last session $\left(t_{3}\right)$. Treatment motivation was assessed at all three time points using the URICA-S [16, 18]. Clinical outcome measures were administered at baseline and after treatment. More specifically, at $t_{1}$ and $t_{3}$, patients completed the SymptomChecklist-90-Revised (SCL-90-R; [26]), Perceived Stress Questionnaire (PSQ; [27]), and Inventory of Interpersonal Problems (IIP; [28]). Additionally, the Structured Clinical Interview, German version (SCID-I, [29, 30]), was administered to all patients at baseline. The initial SCID-I assessment was conducted by one of two $\mathrm{PhD}$-students who had previously completed a university-based SCID training. A university-affiliated expert regularly supervised these students. The local ethics committee of the medical faculty at the University of Tuebingen approved the study protocol (project number 29/2009B02) in compliance with the Helsinki Declaration. All participants provided written informed consent prior to study participation.

\section{Subjects}

Eighty-four inpatients being treated for SSD participated in the study. To be included, subjects had to have received an SCID-I primary diagnosis of a full syndrome of SSD. The general exclusion criteria were as follows: (1) patient age younger than 18 years or older than 59 years, (2) insufficient German language skills, (3) psychotic or substance-related disorders as comorbidities, and (4) current suicidal risk. Because we intended to investigate a naturalistic sample, comorbidities with mental disorders not on the exclusion list were not considered to be exclusions for study participation if SSD was the main treatment diagnosis. Overall, the study sample was $71.4 \%$ female. The average age of participants was 48.0 years (SD 11.9), while $59.5 \%$ were married/with partner, 19\% had an A-level degree and $88.2 \%$ had a formal professional qualification. Furthermore, 50.0\% were employed, $4.8 \%$ were still in job training, $25 \%$ were retired and $20.2 \%$ were unemployed. The sample included $59.5 \%$ with a diagnosis of major depression (36.9\% single episode, $22.6 \%$ recurrent depression) and $10.7 \%$ with an anxiety disorder (9.5\% panic disorder and agoraphobia; $1.2 \%$ specific phobia) as a comorbidity. According to patient interviews and medical data, all patients suffered from SSD symptoms for several years.

\section{Measures \\ URICA-S}

The URICA-S [18] is a short version of the URICA [16]. The URICA-S consists of 16 items that are rated on a scale from 0 (does not apply) to 4 (fully applies) and measures four stages of change (precontemplation, contemplation, action, maintenance) with 4 items per subscale (items listed in the appendix). In a sample of 253 patients, Mander et al. [18] demonstrated an excellent factor structure with factor loadings of $.52 \leq \lambda \leq .87$. Confirmatory factor analyses supported the exploratory model. The instrument yielded acceptable-to-excellent internal consistencies, with $.61 \leq \alpha \leq .84$, along with high correlations with the subscales of the original long-form URICA, with $.83 \leq r \leq .96$. The contemplation, action and maintenance subscales and committed action global score significantly predicted therapeutic outcome. The maintenance score from the URICA-S specifically refers to the struggle with relapse, with stronger negative values indicative of more problems with relapse. Hence, it might be especially important to identify patients at risk of further chronicity. All items of the URICA-S are listed in the online supplement Additional file 1.

\section{$S C L-90-R$}

The SCL-90-R $[26,31]$ is a measure of general symptom severity. It consists of eleven subscales totaling 90 items rated along a 5-point scale. The measure has demonstrated excellent internal consistencies, with $.79 \leq \alpha \leq .89$, and good retest-reliabilities, with $.69 \leq r \leq .92$. Acceptable construct validity has also been reported, with scaleoutcome correlations between $.27 \leq r \leq .81$.

\section{PSQ}

The PSQ [27, 32] is a 30-item scale that was designed to evaluate the stress level of a patient within the seven factors of harassment, overload, irritability, lack of joy, 
fatigue, worries, and tension. Each item is rated along a 5-point scale. The PSQ has demonstrated excellent psychometric properties, with internal consistencies of $.80 \leq \alpha \leq .86$, as well as convergent validity via correlations of $.56 \leq r \leq .73$ with other instruments referring to stress, anxiety, and depression. It has further demonstrated sensitivity to change across the course of therapy.

\section{IIP}

The IIP is a 64-item instrument that utilizes a circumplex structure to assess interpersonal problems [28, 33, 34] . Its eight scales include domineering, intrusive, overly nurturant, exploitable, nonassertive, socially avoidant, cold, and vindictive, rated on a 5-step scale. The IIP has shown excellent psychometric properties, with $.75 \leq \alpha \leq .94$, and criterion validity has been demonstrated through correlations with the SCL-90-R, with $.07 \leq r \leq .75$.

\section{Statistical analyses}

To identify empirical associations between the stages of change and therapeutic outcomes, we initially calculated a set of correlation analyses, computing separate analyses for each of the three therapeutic outcome measures. More specifically, we correlated the URICA-S subscales with the SCL-90-R, PSQ, and IIP global scores in separate analyses. In the next step, we calculated a set of hierarchical regression analyses, computing separate regression analyses for each of the three therapeutic outcome measures as the dependent variable. More specifically, we analyzed one regression model with the SCL-90- $\mathrm{R}$ global score at $t_{3}$, one with the PSQ global score at $t_{3}$ and one with the IIP global score at $t_{3}$ as the dependent variable. In each model, we blockwise entered the four URICA-S subscale scores as well as the baseline score of the relevant outcome measure as predictors. In the first block, we entered the global score of the relevant outcome measure at baseline as an autoregressor. In the second block, we always entered the maintenance score because it demonstrated the strongest association with the therapeutic outcome in the correlation analyses. The order in which the other subscales were entered in subsequent blocks depended on the strengths of their correlations with the outcome measures.

\section{Results}

Correlation analyses of the URICA-S revealed the following results for the stages of change subscales: Precontemplation was not significantly associated with any of the therapeutic outcome scores. Contemplation at $t_{1}$ was significantly associated with the PSQ, but no other significant associations emerged for contemplation. Action was not significantly associated with any of the therapeutic outcome scores. Maintenance was significantly associated with all three outcome measures (the SCL-
90-R, the PSQ and the IIP) at all three measuring times. The results of the correlation analyses are depicted in Table 1.

In the multiple regression analyses, only maintenance proved to be a significant predictor of outcome when controlling for the baseline outcome scores.

Concerning SCL-90- $\mathrm{R}$, maintenance at $\mathrm{t}_{1}$ was a significant predictor of SCL-90- $\mathrm{R}$ at $\mathrm{t}_{3}$ while controlling for SCL-90-R at $\mathrm{t}_{1}, \Delta R^{2}=.08, p=.005$. Maintenance at $\mathrm{t}_{2}$ and $t_{3}$ did not significantly predict SCL-90-R at $t_{3}$. However, trends $(p<.10)$ were observable, with $\Delta R^{2}=.05$. $p=.065$ for maintenance at $\mathrm{t}_{2}$ and $\Delta R^{2}=.022, p=.069$ for maintenance at $\mathrm{t}_{3}$.

Concerning the PSQ, maintenance was a significant predictor at all three measuring times. More specifically, the maintenance score was a significant predictor of the PSQ at $t_{3}$ while controlling for the PSQ at $t_{1}$; with $\Delta R^{2}=.08, p<.001$ for maintenance at $\mathrm{t}_{1} ; \Delta R^{2}=.06$, $p=.022$ for maintenance at $\mathrm{t}_{2}$; and $\Delta R^{2}=.07, p=.017$ for maintenance at $t_{3}$.

Concerning the IIP, maintenance at $t_{1}$ and $t_{2}$ were significant predictors. More specifically, the maintenance score was a significant predictor of the IIP at $t_{3}$ while controlling for the IIP at $\mathrm{t}_{1}$, with $\Delta R^{2}=.02, p=.023$ for maintenance at $\mathrm{t}_{1}$; and with $\Delta R^{2}=.03, p=.034$ for maintenance at $t_{2}$. Maintenance at $t_{3}$ was not a significant predictor of the IIP at $t_{3}$. However, a trend $(p<.10)$ was observable, with $\Delta R^{2}=.02, p=.087$.

None of the other stages of change proved to be a significant predictor in any regression model. For parsimonious reasons, we only report the significant regression models in Tables 2, 3 and 4.

Table 1 Correlations of the URICA subscales at all three measuring times with SCL, PSQ and IIP at the end of therapy

\begin{tabular}{|c|c|c|c|c|}
\hline & Precontemplation & Contemplation & Action & Maintenance \\
\hline \multicolumn{5}{|c|}{ SCL-90-R } \\
\hline$t_{1}$ & .11 & .15 & .03 & $.48^{* *}$ \\
\hline$t_{2}$ & .13 & .12 & -.07 & $.38^{* *}$ \\
\hline$t_{3}$ & .00 & .22 & -.04 & $.43^{* *}$ \\
\hline \multicolumn{5}{|l|}{ PSQ } \\
\hline$t_{1}$ & .08 & $.28^{*}$ & .19 & $.48^{* *}$ \\
\hline$t_{2}$ & .02 & -.05 & -.34 & $.34^{* *}$ \\
\hline$t_{3}$ & .15 & .17 & -.03 & $.38^{* *}$ \\
\hline \multicolumn{5}{|l|}{ IIP } \\
\hline$t_{1}$ & .11 & .18 & .06 & $.42^{* *}$ \\
\hline$t_{2}$ & .07 & .00 & -.04 & $.32^{*}$ \\
\hline$t_{3}$ & -.04 & .09 & -.14 & $.30^{*}$ \\
\hline
\end{tabular}

$\mathrm{t}_{1} / \mathrm{t}_{2} / \mathrm{t}_{3}=$ at baseline/8th/last therapy session

SCL-90-R Symptom-Checklist-90-Revised, PSQ Perceived Stress Questionnaire, IIP Inventory of Interpersonal Problems

${ }^{*}=p<.05 ;{ }^{* *}=p<.01$ 
Table 2 Significant linear regression coefficients of stages of change predicting SCL-90 symptomatology at the end of therapy

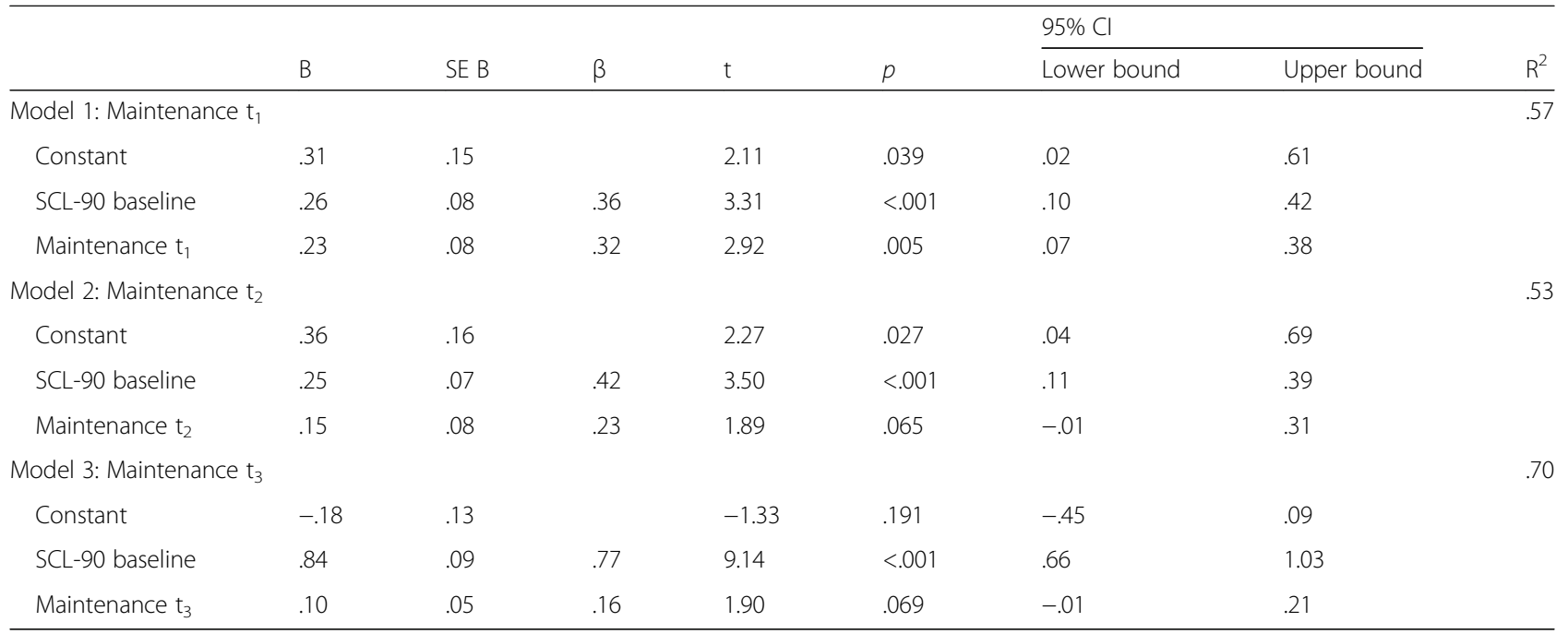

$t_{1} / t_{2} / t_{3}=$ at baseline/after 8th therapy session/ end of therapy. Model $1 /$ Model 2 / Model $3=$ significant stages of change predictors at baseline/8th/last therapy session for SCL-90 at the end of therapy.

SCL-90-R Symptom-Checklist-90-Revised

\section{Discussion}

SSD patients show disproportionately elevated rates of medical care utilization, leading to high health care costs [7]. Further, therapeutic intervention programs for SSD patients show only small-to-moderate effect sizes $[7,8]$. This is partly explained by the ambivalent motivation of SSD patients to receive psychological treatments [10]. Hence, fostering treatment motivation could increase treatment success in this patient group [8]. The aim of the present study was to investigate associations between the TTM stages of change and general symptomatology and general stress level, as well as interpersonal functioning in SSD patients. We specifically hypothesized that higher scores on the URICA-S maintenance scale, as an indicator of motivational relapse struggle, would be associated with more negative treatment outcomes in SSD inpatients. Of note, our results indicate that URICA-S maintenance at the first measuring time was a significant predictor of all three outcome measures. This applied to both the correlation analyses and longitudinal multiple regression analyses controlling for baseline symptom severity. These findings indicate that the higher the maintenance score at the beginning of therapy, that is, the greater the struggle with relapse, the

Table 3 Significant linear regression coefficients of stages of change predicting PSQ symptomatology at the end of therapy

\begin{tabular}{|c|c|c|c|c|c|c|c|c|}
\hline & & & & & & $95 \% \mathrm{Cl}$ & & \\
\hline & B & SE B & $\beta$ & $\mathrm{t}$ & $p$ & Lower bound & Upper bound & $R^{2}$ \\
\hline Model 1: Maintenar & & & & & & & & .49 \\
\hline Constant & .06 & .06 & & .92 & .362 & -.07 & .18 & \\
\hline PSQ baseline & .61 & .11 & .52 & 5.68 & $<.001$ & .40 & .83 & \\
\hline Maintenance $t_{1}$ & .05 & .02 & .31 & 3.33 & $<.001$ & .02 & .08 & \\
\hline Model 2: Maintenar & & & & & & & & .43 \\
\hline Constant & .03 & .07 & & .33 & .74 & -.12 & .17 & \\
\hline PSQ baseline & .67 & .12 & .57 & 5.60 & $<.001$ & .43 & .91 & \\
\hline Maintenance $t_{2}$ & .04 & .02 & .24 & 2.35 & .022 & .006 & .08 & \\
\hline Model 3: Maintenar & & & & & & & & .42 \\
\hline Constant & .02 & .08 & & .26 & .79 & -.15 & .19 & \\
\hline PSQ baseline & .67 & .14 & .54 & 4.94 & $<.001$ & .40 & .95 & \\
\hline Maintenance $t_{3}$ & .04 & .02 & .27 & 2.46 & .017 & .01 & .08 & \\
\hline
\end{tabular}

$t_{1} / t_{2} / t_{3}=$ at baseline/after 8 th therapy session/ end of therapy. Model 1 / Model 2 / Model 3 = significant stages of change predictors at baseline/8th/last therapy session for PSQ at the end of therapy. PSQ Perceived Stress Questionnaire 
Table 4 Significant linear regression coefficients of stages of change predicting IIP symptomatology at the end of therapy

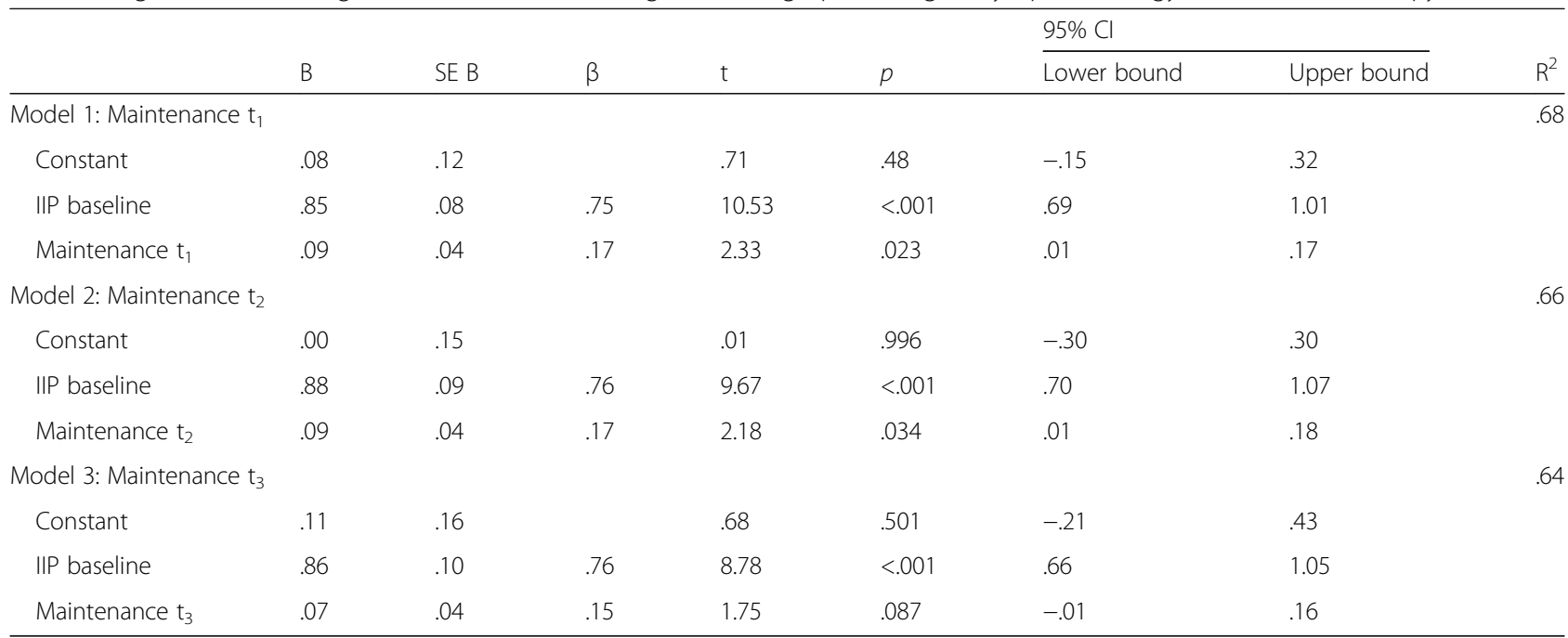

$t_{1} / t_{2} / t_{3}=$ at baseline/after 8 th therapy session/ end of therapy. Model $1 /$ Model $2 /$ Model 3 = significant stages of change predictors at baseline/8th/last therapy session for IIP at the end of therapy.

IIP Inventory of Interpersonal Problems

lower the change in the three outcome measures across the course of therapy. This is clinically relevant, as it indicates that assessment of the patients' maintenance stage of change at the beginning of therapy can help identify patients who will be more likely to have negative treatment outcomes. Furthermore, URICA-S maintenance at therapy admission significantly predicted patients' general clinical symptoms, general stress levels and interpersonal functioning. Consequently, the results indicate that URICA-S maintenance, as assessed early in treatment, is an important predictor of three essential aspects of the general functioning of SSD patients.

However, an important limitation of our results is that maintenance assessed at $t_{2}$ and $t_{3}$ did not significantly predict general symptom severity as assessed by the SCL-90-R or interpersonal functioning as assessed by the IIP. Here, only trends $(p<.10)$ toward predictive effects were observable. These effects could possibly reach significance with more statistical power in larger SSD samples. However, identifying this aspect empirically remains a task for future research. Hence, given our current research results, it remains unclear whether maintenance assessed later in therapy is still a relevant predictor of the therapeutic outcome. Consequently, our results must be regarded as preliminary and should be interpreted with caution. Further, and similar to our previous findings in AN patients [21], no other stages of change were significant predictors of outcome.

Hence, in SSD patients, the maintenance score assessed at therapy admission seems to be an indicator of the relapse threat, which could be helpful for early identification of inpatients who could improve during therapy as well as those who might suffer from repeated drawbacks. In many SSD patients, the course of their disorder is characterized by high chronicity and recurrent relapses, while the first months after inpatient treatment seem to represent a highly vulnerable phase $[7,8]$. Consequently, should our results be replicated in future studies, the URICA-S maintenance ratings could be used to supplement the clinical judgments of expert diagnosticians to identify high-risk patient subgroups that need further treatment elements in addition to routine therapy. More specifically, similar to preliminary studies in AN patients [22], these patient subgroups could receive a specific treatment to address ambivalence, skills training related to drawbacks, and additional psychoeducation as well as aftercare programs following inpatient treatment.

As mentioned previously, the PSOCQ can also be a disorder-specific stage of change measure for SSD patients, and several studies have documented its clinical utility (e.g. [10, 24]). Future research should use the PSOCQ to examine the extent to which our current study results can be replicated with a disorder-specific measure. In contrast to the URICA-S, where maintenance refers to relapse struggle, the PSOCQ maintenance scale reflects the ability to maintain progress [23, 24]. The current study results indicate that a relapse measure is important in SSD research, as high chronicity and recurrent relapses are important characteristics of the disorder $[7,8]$. Consequently, it might be of interest to devise a new, more differentiated disorder-specific maintenance scale for the PSOCQ that contains a progress maintenance component and relapse facet.

The current study has the following limitations: First, our study was conducted in an inpatient setting. Hence, the generalizability of the effects to other treatment 
setting remains unclear and should be addressed in future studies. Second, we conducted a naturalistic study without a control group. Future research should try to directly implement motivational interventions to improve the outcomes of patients who score high for URICA-S maintenance at the beginning of therapy. Consequently, a randomized controlled design, such as one that incorporates motivational interviewing [35] in the experimental condition and typical treatment in the control condition, should be applied. Third, two predictors in regression analyses were only marginally significant. Hence, our results should be interpreted with caution. However, when interpreting the results, not only should the specific effect of the single predictor be recognized but the whole pattern of results should be interpreted. More specifically, maintenance was a significant predictor of therapeutic outcome irrespective of measuring time and whether general clinical symptoms, the general stress level or interpersonal functioning were entered as dependent variables. Consequently, maintenance seems to be one important aspect that should be addressed to increase the chance of successful psychotherapy. Fourth, in the current study, approximately $60 \%$ of patients suffered from comorbid depressive disorders. SSD patients with and without depression might show different patterns regarding the effects of treatment motivation on the therapeutic outcome. In our study, the statistical power to identify subgroup effects of SSD patients with and without comorbidities was too low. Hence, future studies should investigate this aspect in more adequately powered subsamples. Finally, future studies should investigate associations between motivation and outcome on a session-to-session basis, as this could capture greater variations and nuances within the therapeutic process.

\section{Conclusions}

In conclusion, the present study analyzed associations between the stages of change assessed with the URICA$\mathrm{S}$ and therapeutic outcome in SSD patients. The URICA-S maintenance score assessed at the beginning of therapy could be used to help identify SSD patients who have a relapse risk. Future studies should incorporate randomized controlled trials to investigate whether this subgroup could benefit from motivational interventions to address relapse.

\section{Additional file}

Additional file 1: Items_of_the_URICA-S, the additional file includes all items of the URICA-S scale. (DOCX $12 \mathrm{~kb}$ )

\section{Acknowledgements}

We would like to thank the health professionals of the Department of Psychosomatic Medicine at the University of Tübingen.

\section{Funding}

This study was partly supported by the German Research Foundation (DFG MA6526/2-1 and DFG JU 2998/1-1) and by the Köhler Foundation (S112/10145/ 10). We acknowledge financial support by Deutsche Forschungsgemeinschaft and Ruprecht-Karls-Universität Heidelberg within the funding programme Open Access Publishing.

\section{Availability of data and materials}

The datasets used and/or analysed during the current study are available from the corresponding author on reasonable request.

\section{Authors' contributions}

JM drafted the manuscript. GS, UD and FJ delivered feedback on a first version of the paper. GS, HB, UD, SZ and FJ critically revised the second version of the manuscript for important intellectual content. JM, FJ and SZ contributed to the design of the study. All authors have approved the final version of the manuscript.

\section{Authors' information}

Non applicable.

\section{Ethics approval and consent to participate}

The local ethics committee (ethics committee of the medical faculty of the University of Tübingen, project numer 29/2009B02) approved the study protocol in accordance with the Helsinki Declaration. All participants provided written, informed consent.

\section{Consent for publication}

Non applicable.

\section{Competing interests}

The authors declare that they have no competing interests.

\section{Publisher's note}

Springer Nature remains neutral with regard to jurisdictional claims in published maps and institutional affiliations.

\section{Author details \\ ${ }^{1}$ Center for Psychological Psychotherapy, University of Heidelberg, Heidelberg, Germany. ${ }^{2}$ Department of Psychosomatic Medicine and Psychotherapy, Medical University Hospital of Tübingen, Tübingen, Germany. ${ }^{3}$ Department of Psychosomatic Medicine and Psychotherapy, Medical University Hospital of Heidelberg, Heidelberg, Germany. ${ }^{4}$ Bergheimer Str. 58a,} 69115, Heidelberg, Germany.

Received: 15 February 2017 Accepted: 26 June 2017

Published online: 03 July 2017

References

1. American Psychological Association. Diagnostic and statistical Manual of mental disorders - DSM-5. 5th ed. Washington, D.C.: American Psychiatric Publishing; 2013.

2. Rief W, Rojas G. Stability of somatoform symptoms-implications for classification. Psychosom Med. 2007;69(9):864-9.

3. Jackson $\mathrm{JL}$, Kroenke K. Prevalence, impact, and prognosis of multisomatoform disorder in primary care: a 5-year follow-up study. Psychosom Med. 2008;70(4):430-4.

4. de Waal MWM, Arnold IA, Eekhof JAH, van Hemert AM. Somatoform disorders in general practice: prevalence, functional impairment and comorbidity with anxiety and depressive disorders. Br J Psychiatry. 2004;184(6):470-6.

5. Gottschalk J-M, Bleichhardt G, Kleinstäuber M, Berking M, Rief W. Erweiterung der kognitiven verhaltenstherapie um emotionsregulationstraining bei patienten mit multiplen somatoformen symptomen: Ergebnisse einer kontrollierten pilotstudie. = extension of CBT by emotion regulation training in patients with multiple somatoform symptoms. Verhaltenstherapie. 2015;25(1):13-21.

6. Martin A, Rief W. Relevance of cognitive and behavioral factors in medically unexplained syndromes and somatoform disorders. Psychiatr Clin N Am. 2011;34(3):565-78. 
7. Kleinstäuber M, Witthoft M, Hiller W. Efficacy of short-term psychotherapy for multiple medically unexplained physical symptoms: a meta-analysis. Clin Psychol Rev. 2011;31(1):146-60.

8. van Dessel $\mathrm{N}$, den Boeft M, van der Wouden JC, Kleinstauber M, Leone SS, Terluin B, Numans ME, van der Horst HE, van Marwijk H. Nonpharmacological interventions for somatoform disorders and medically unexplained physical symptoms (MUPS) in adults. The Cochrane database of systematic reviews. 2014;11:Cd011142.

9. Kleinstäuber M, Gottschalk J, Berking M, Rau J, Rief W. Enriching cognitive behavior therapy with emotion regulation training for patients with multiple medically unexplained symptoms (ENCERT): design and implementation of a multicenter, randomized, active-controlled trial. Contemporary clinical trials. 2016:47:54-63.

10. Heider J, Kock K, Sehlbrede M, Schroder A. Readiness to change as a moderator of therapy outcome in patients with somatoform disorders. Psychotherapy research: journal of the Society for Psychotherapy Research. 2017:1-12.

11. Prochaska JO, Norcross JC. Systems of psychotherapy: a transtheoretical analysis 8edn. Belmont, CA US: Thomson Brooks/Cole Publishing Co; 2013.

12. Prochaska JO, DiClemente CC. Transtheoretical therapy: toward a more integrative model of change. Psychotherapy: Theory, Research \& Practice. 1982;19(3):276-88

13. Norcross JC, Krebs PM, Prochaska JO. Stages of change. J Clin Psychol. 2011; 67(2):143-54.

14. Prochaska JO, DiClemente CC. Stages and processes of self-change of smoking: toward an integrative model of change. J Consult Clin Psychol. 1983;51(3):390-5.

15. Mander J, Wittorf A, Klingberg S, Teufel M, Zipfel S, Sammet I. The patient perspective on therapeutic change: the investigation of associations between stages of change and general mechanisms of change in psychotherapy research. J Psychother Integr. 2014;24(2):122-37.

16. McConnaughy EA, Prochaska JO, Velicer WF. Stages of change in psychotherapy: measurement and sample profiles. Psychotherapy: Theory, Research \& Practice. 1983;20(3):368-75.

17. Maurischat C. Erfassung der 'Stages of Change' im Transtheoretischen Modell-eine Übersicht dimensionaler versus kategorialer Ansätze [assessment of the "stages of change" using the Transtheoretical model an overview of dimensional versus categorical approaches]. Z Klin Psychol Psychiatr Psychother. 2002;50(4):343-67.

18. Mander J, Wittorf A, Teufel M, Schlarb A, Hautzinger M, Zipfel S, Sammet I. Patients with depression, somatoform disorders, and eating disorders on the stages of change: validation of a short version of the URICA. Psychotherapy. 2012;49(4):519-27.

19. Junne F, Ziser K, Mander J, Martus P, Denzer C, Reinehr T, Wabitsch M, Wiegand S, Renner T, Giel KE, et al. Development and psychometric validation of the 'Parent perspective University of Rhode Island Change Assessment-Short' (PURICA-S) Questionnaire for the application in parents of children with overweight and obesity. BMJ Open. 2016;6(11):e012711.

20. Carbonari JP, DiClemente CC. Using transtheoretical model profiles to differentiate levels of alcohol abstinence success. J Consult Clin Psychol. 2000;68(5):810-7.

21. Mander J, Teufel M, Keifenheim K, Zipfel S, Giel KE. Stages of change, treatment outcome and therapeutic alliance in adult inpatients with chronic anorexia nervosa. BMC Psychiatry. 2013;13:111.

22. Giel KE, Leehr EJ, Becker S, Herzog W, Junne F, Schmidt U, Zipfel S. Relapse prevention via videoconference for anorexia nervosa - findings from the RESTART pilot study. Psychother Psychosom. 2015;84(6):381-3.

23. Kerns RD, Rosenberg R, Jamison RN, Caudill MA, Haythornthwaite J. Readiness to adopt a self-management approach to chronic pain: the pain stages of change Questionnaire (PSOCQ). Pain. 1997;72(1-2):227-34.

24. Maurischat C, Härter M, Bengel J. Der Freiburger Fragebogen - Stadien der Bewältigung chronischer Schmerzen (FF-STABS): Faktorenstruktur, psychometrische Eigenschaften und Konstruktvalidierung. [The Freiburg Questionnaire - Stages of Chronic Pain Management (FQ-STAPM): Factoria structure, psychometric properties and construct validation]. Diagnostica. 2002:48(4):190-9.

25. Maurischat $C$, Härter $M$, Kerns RD, Bengel J. Further support for the pain stages of change model: suggestions for improved measurement. Eur J Pain. 2006;10(1):41-9.

26. Derogatis LR, Fitzpatrick M: The SCL-90-R, the Brief Symptom Inventory (BSI), and the BSI-18. In: The use of psychological testing for treatment planning and outcomes assessment: Volume 3: Instruments for adults (3rd ed). edn. Edited by Maruish ME. Mahwah, NJ US: Lawrence Erlbaum Associates Publishers; 2004: 1-41.

27. Levenstein S, Prantera C, Varvo V, Scribano ML, Berto E, Luzi C, Andreoli A. Development of the Perceived stress Questionnaire: a new tool for psychosomatic research. J Psychosom Res. 1993;37(1):19-32.

28. Horowitz LM, Rosenberg SE, Baer BA, Ureño G, Villaseñor VS. Inventory of interpersonal problems: psychometric properties and clinical applications. J Consult Clin Psychol. 1988;56(6):885-92.

29. Wittchen HU, Wunderlich U, Gruschwitz S, Zaudig M. Strukturiertes Klinisches Interview für DSM-IV (SKID I) [the structured clinical Interview for DSM-IV (SCID I)]. Göttingen, Germany: Hogrefe; 1997

30. American Psychiatric A. Falkai P, Wittchen H-U, Döpfner M, Gaebel W, Maier W, Rief W, Saß: Diagnostisches und Statistisches Manual Psychischer Störungen DSM-5 [Diagnostic and Statistical Manual of Mental Disorders DSM-5]. Göttingen: Hogrefe; 2015.

31. Franke GH. Die Symptom-Checkliste von L. R. Derogatis (1977) - Deutsche Version [Symptom Check List 90 Items Revised (Derogatis, L.R., 1977) German version]. Beltz Test GmbH: Göttingen; 2002.

32. Fliege $H$, Rose M, Arck P, Levenstein S, Klapp BF. Validierung des 'Perceived Stress Questionnaire' (PSQ) an einer deutschen Stichprobe. Diagnostica. 2001:47(3):142-52

33. Brähler E, Horowitz LM, Kordy H, Schumacher J, Strauß B. Zur Validierung des Inventars zur Erfassung Interpersonaler Probleme (IIP)—Ergebnisse einer Repräsentativbefragung in Ost- und Westdeutschland [validation of the Inventory of interpersonal problems - results of a Represantative investigation in east- and West-Germany]. Psychother Psychosom Med Psychol. 1999:49(11):422-31.

34. Horowitz LM. The study of interpersonal problems: a Leary legacy. J Pers Assess. 1996:66(2):283-300

35. Miller W, Rollnick S. Motivational interviewing. Preparing people to change addictive behaviour. New York: Guildford Press; 1991

\section{Submit your next manuscript to BioMed Central and we will help you at every step:}

- We accept pre-submission inquiries

- Our selector tool helps you to find the most relevant journal

- We provide round the clock customer support

- Convenient online submission

- Thorough peer review

- Inclusion in PubMed and all major indexing services

- Maximum visibility for your research

Submit your manuscript at www.biomedcentral.com/submit
) Biomed Central 